\title{
Existence solutions for boundary value problem of nonlinear fractional $q$-difference equations
}

\author{
Wen-Xue Zhou ${ }^{1,2^{*}}$ and Hai-Zhong Liu'
}

\section{"Correspondence:}

wxzhou2006@126.com

'Department of Mathematics,

Lanzhou Jiaotong University,

Lanzhou, 730070, P.R. China

${ }^{2}$ School of Mathematical Sciences,

Fudan University, Shanghai, 200433,

P.R. China

\section{Springer}

\begin{abstract}
In this paper, we discuss the existence of weak solutions for a nonlinear boundary value problem of fractional $q$-difference equations in Banach space. Our analysis relies on the Mönch's fixed-point theorem combined with the technique of measures of weak noncompactness.
\end{abstract}

MSC: $26 \mathrm{~A} 33 ; 34 \mathrm{~B} 15$

Keywords: boundary value problem; fractional q-difference equations; Caputo fractional derivative; weak solutions

\section{Introduction}

Fractional differential calculus is a discipline to which many researchers are dedicating their time, perhaps because of its demonstrated applications in various fields of science and engineering [1]. Many researchers studied the existence of solutions to fractional boundary value problems, for example, [2-10].

The $q$-difference calculus or quantum calculus is an old subject that was initially developed by Jackson [11, 12]; basic definitions and properties of $q$-difference calculus can be found in $[13,14]$.

The fractional $q$-difference calculus had its origin in the works by Al-Salam [15] and Agarwal [16]. More recently, maybe due to the explosion in research within the fractional differential calculus setting, new developments in this theory of fractional $q$-difference calculus were made, for example, $q$-analogues of the integral and differential fractional operators properties such as Mittage-Leffler function [17], just to mention some.

El-Shahed and Hassan [18] studied the existence of positive solutions of the $q$-difference boundary value problem:

$$
\left\{\begin{array}{l}
-D_{q}^{2} u(t)=a(t) f(u(t)), \quad t \in J:=[0,1], \\
\alpha u(0)-\beta D_{q} u(0)=0, \quad \gamma u(1)+\delta D_{q} u(1)=0 .
\end{array}\right.
$$

Ferreira [19] considered the existence of positive solutions to nonlinear $q$-difference boundary value problem:

$$
\left\{\begin{array}{l}
\left({ }_{R L} D_{q}^{\alpha} u\right)(t)=-f(t, u(t)), \quad 0<t<1,1<\alpha \leq 2, \\
u(0)=u(1)=0 .
\end{array}\right.
$$

( 2013 Zhou and Liu; licensee Springer. This is an Open Access article distributed under the terms of the Creative Commons Attribution License (http://creativecommons.org/licenses/by/2.0), which permits unrestricted use, distribution, and reproduction in any medium, provided the original work is properly cited. 
Ferreira [20] studied the existence of positive solutions to nonlinear $q$-difference boundary value problem:

$$
\begin{cases}\left({ }_{R L} D_{q}^{\alpha} u\right)(t)=-f(t, u(t)), & 0<t<1,2<\alpha \leq 3, \\ u(0)=\left(D_{q} u\right)(0)=0, & \left(D_{q} u\right)(1)=\beta \geq 0 .\end{cases}
$$

El-Shahed and Al-Askar [21] studied the existence of positive solutions to nonlinear q-difference equation:

$$
\begin{cases}{ }_{C} D_{q}^{\alpha} u+a(t) f(u(t))=0, & 0<t<1,2<\alpha \leq 3, \\ u(0)=\left(D_{q}^{2} u\right)(0)=0, & \gamma\left(D_{q} u\right)(1)+\beta D_{q}^{2} u(1)=0\end{cases}
$$

where $\gamma, \beta \geq 0$ and ${ }_{C} D_{q}$ is the fractional $q$-derivative of the Caputo type.

Ahmad, Alsaedi and Ntouyas [22] discussed the existence of solutions for the secondorder $q$-difference equation with nonseparated boundary conditions

$$
\left\{\begin{array}{l}
D_{q}^{2} u(t)=f(t, u(t)), \quad t \in I \\
u(0)=\eta u(T), \quad D_{q} u(0)=\eta D_{q} u(T)
\end{array}\right.
$$

where $f \in C(I \times \mathbb{R}, \mathbb{R}), I=[0, T] \cap q^{\overline{\mathbb{N}}}, q^{\overline{\mathbb{N}}}:=\left\{q^{n}: n \in \mathbb{N}\right\} \cup\{0\}$, and $T \in q^{\overline{\mathbb{N}}}$ is a fixed constant, and $\eta \neq 1$ is a fixed real number.

Ahmad and Nieto [23] discussed a nonlocal nonlinear boundary value problem (BVP) of third-order $q$-difference equations given by

$$
\left\{\begin{array}{l}
D_{q}^{3} u(t)=f(t, u(t)), \quad t \in I_{q}, \\
u(0)=0, \quad D_{q} u(0)=0, \quad u(1)=\alpha u(\eta)
\end{array}\right.
$$

where $f \in C\left(I_{q} \times \mathbb{R}, \mathbb{R}\right), I_{q}=\{0,1\} \cup\left\{q^{n}: n \in \mathbb{N}\right\}$, and $q \in(0,1)$ is a fixed constant, $\eta \in\left\{q^{n}\right.$ : $n \in \mathbb{N}\}$ and $\alpha \neq 1 / \eta^{2}$ is a real number.

This paper is mainly concerned with the existence results for the following fractional q-difference equations:

$$
\left\{\begin{array}{l}
{ }_{C} D_{q}^{\alpha} u+f(t, u)=0, \quad t \in J=[0,1], 2<\alpha \leq 3 \\
u(0)=\left(D_{q}^{2} u\right)(0)=0, \quad \gamma\left(D_{q} u\right)(1)+\beta D_{q}^{2} u(1)=0,
\end{array}\right.
$$

where $\gamma, \beta \geq 0$ and ${ }_{C} D_{q}$ is the fractional $q$-derivative of the Caputo type. $f: J \times E \rightarrow E$ is a given function satisfying some assumptions that will be specified later, and $E$ is a Banach space with norm $\|u\|$.

To investigate the existence of solutions of the problem above, we use Mönch's fixedpoint theorem combined with the technique of measures of weak noncompactness, which is an important method for seeking solutions of differential equations. This technique was mainly initiated in the monograph of Banaś and Goebel [24], and subsequently developed and used in many papers; see, for example, Banaś et al. [25], Guo et al. [26], Krzyska and Kubiaczyk [27], Lakshmikantham and Leela [28], Mönch [29], O’Regan [30, 31], Szufla 
$[32,33]$ and the references therein. As far as we know, there are very few results devoted to weak solutions of nonlinear fractional differential equations [34-38]. Motivated by the above mentioned papers, the purpose of this paper is to establish the existence results for the boundary value problem (1.1) by virtue of the Mönch's fixed-point theorem combined with the technique of measures of weak noncompactness.

The remainder of this article is organized as follows. In Section 2, we provide some basic definitions, preliminaries facts and various lemmas, which are needed later. In Section 3, we give main results of the problem (1.1). In the end, we also give an example for the illustration of the theories established in this paper.

\section{Preliminaries and lemmas}

In this section, we present some basic notations, definitions and preliminary results, which will be used throughout this paper.

Let $q \in(0,1)$ and define $[13]$

$$
[a]_{q}=\frac{q^{a}-1}{q-1}=a^{a-1}+\cdots+1, \quad a \in \Re .
$$

The $q$-analogue of the power $(a-b)^{n}$ is

$$
(a-b)^{(0)}=1, \quad(a-b)^{(n)}=\prod_{k=0}^{n-1}\left(a-b q^{k}\right), \quad a, b \in \Re, n \in \mathbb{N}
$$

If $\alpha$ is not a positive integer, then

$$
(a-b)^{(\alpha)}=a^{\alpha} \prod_{i=0}^{\infty} \frac{\left(1-(b / a) q^{i}\right)}{\left(1-(b / a) q^{\alpha+i}\right)}
$$

Note that if $b=0$, then $a^{(\alpha)}=a^{\alpha}$. The $q$-gamma function is defined by

$$
\Gamma_{q}(x)=\frac{(1-q)^{(x-1)}}{(1-q)^{x-1}}, \quad x \in \mathfrak{R} \backslash\{0,-1,-2, \ldots\}, 0<q<1,
$$

and satisfies $\Gamma_{q}(x+1)=[x]_{q} \Gamma_{q}(x)$.

The $q$-derivative of a function $f$ is here defined by

$$
D_{q} f(x)=\frac{d_{q} f(x)}{d_{q} x}=\frac{f(q x)-f(x)}{(q-1) x}
$$

and $q$-derivatives of higher order by

$$
D_{q}^{n} f(x)= \begin{cases}f(x), & \text { if } n=0 \\ D_{q} D_{q}^{n-1} f(x), & \text { if } n \in \mathbb{N} .\end{cases}
$$

The $q$-integral of a function $f$ defined in the interval $[0, b]$ is given by

$$
\int_{0}^{x} f(t) d_{q} t=x(1-q) \sum_{n=0}^{\infty} f\left(x q^{n}\right) q^{n}, \quad 0 \leq|q|<1, x \in[0, b] .
$$


If $a \in[0, b]$ and $f$ is defined in the interval $[0, b]$, its integral from $a$ to $b$ is defined by

$$
\int_{a}^{b} f(t) d_{q} t=\int_{0}^{b} f(t) d_{q} t-\int_{0}^{a} f(t) d_{q} t .
$$

Similarly, as done for derivatives, an operator $I_{q}^{n}$ can be defined, namely,

$$
\left(I_{q}^{0} f\right)(x)=f(x), \quad\left(I_{q}^{n} f\right)(x)=I_{q}\left(I_{q}^{n-1} f\right)(x), \quad n \in \mathbb{N} .
$$

The fundamental theorem of calculus applies to these operators $I_{q}$ and $D_{q}$, that is,

$$
\left(D_{q} I_{q} f\right)(x)=f(x)
$$

and if $f$ is continuous at $x=0$, then

$$
\left(I_{q} D_{q} f\right)(x)=f(x)-f(0)
$$

Basic properties of the two operators can be found in the book mentioned in [13]. We now point out three formulas that will be used later $\left({ }_{i} D_{q}\right.$ denotes the derivative with respect to variable $i$ ) [19]

$$
\begin{aligned}
& {[a(t-s)]^{(\alpha)}=a^{\alpha}(t-s)^{(\alpha)},} \\
& { }_{t} D_{q}(t-s)^{(\alpha)}=[\alpha]_{q}(t-s)^{(\alpha-1)}, \\
& \left({ }_{x} D_{q} \int_{0}^{x} f(x, t) d_{q} t\right)(x)=\int_{0}^{x}{ }_{x} D_{q} f(x, t) d_{q} t+f(q x, x) .
\end{aligned}
$$

Remark 2.1 We note that if $\alpha>0$ and $a \leq b \leq t$, then $(t-a)^{(\alpha)} \geq(t-b)^{(\alpha)}$ [19].

Let $J:=[0,1]$ and $L^{1}(J, E)$ denote the Banach space of real-valued Lebesgue integrable functions on the interval $J, L^{\infty}(J, E)$ denote the Banach space of real-valued essentially bounded and measurable functions defined over $J$ with the norm $\|\cdot\|_{L^{\infty}}$.

Let $E$ be a real reflexive Banach space with norm $\|\cdot\|$ and dual $E^{*}$, and let $(E, \omega)=$ $\left(E, \sigma\left(E, E^{*}\right)\right)$ denote the space $E$ with its weak topology. Here, $C(J, E)$ is the Banach space of continuous functions $x: J \rightarrow E$ with the usual supremum norm $\|x\|_{\infty}:=\sup \{\|x(t)\|: t \in J\}$.

Moreover, for a given set $V$ of functions $v: J \mapsto \mathbb{R}$, let us denote by $V(t)=\{v(t): v \in$ $V\}, t \in J$, and $V(J)=\{v(t): v \in V, t \in J\}$.

Definition 2.1 A function $h: E \rightarrow E$ is said to be weakly sequentially continuous if $h$ takes each weakly convergent sequence in $E$ to a weakly convergent sequence in $E$ (i.e. for any $\left(x_{n}\right)_{n}$ in $E$ with $x_{n}(t) \rightarrow x(t)$ in $(E, \omega)$ then $h\left(x_{n}(t)\right) \rightarrow h(x(t))$ in $(E, \omega)$ for each $\left.t \rightarrow J\right)$.

Definition 2.2 [39] The function $x: J \rightarrow E$ is said to be Pettis integrable on $J$ if and only if there is an element $x_{J} \in E$ corresponding to each $I \subset J$ such that $\varphi\left(x_{I}\right)=\int_{I} \varphi(x(s)) d s$ for all $\varphi \in E^{*}$, where the integral on the right is supposed to exist in the sense of Lebesgue. By definition, $x_{I}=\int_{I} x(s) d s$.

Let $P(J, E)$ be the space of all $E$-valued Pettis integrable functions in the interval $J$. 
Lemma 2.1 [39] If $x(\cdot)$ is Pettis integrable and $h(\cdot)$ is a measurable and an essentially bounded real-valued function, then $x(\cdot) h(\cdot)$ is Pettis integrable.

Definition 2.3 [40] Let $E$ be a Banach space, $\Omega_{E}$ the set of all bounded subsets of $E$, and $B_{1}$ the unit ball in $E$. The De Blasi measure of weak noncompactness is the map $\beta: \Omega_{E} \rightarrow$ $[0, \infty)$ defined by

$$
\begin{aligned}
\beta(X)= & \inf \{\epsilon>0: \text { there exists a weakly compact subset } \Omega \text { of } E \\
& \text { such that } \left.X \subset \epsilon B_{1}+\Omega\right\} .
\end{aligned}
$$

Lemma 2.2 [40] The De Blasi measure of noncompactness satisfies the following properties:

(a) $S \subset T \Rightarrow \beta(S) \leq \beta(T)$;

(b) $\beta(S)=0 \Leftrightarrow S$ is relatively weakly compact;

(c) $\beta(S \cup T)=\max \{\beta(S), \beta(T)\}$;

(d) $\beta\left(\bar{S}^{\omega}\right)=\beta(S)$, where $\bar{S}^{\omega}$ denotes the weak closure of $S$;

(e) $\beta(S+T) \leq \beta(S)+\beta(T)$;

(f) $\beta(a S)=|a| \alpha(S)$;

(g) $\beta(\operatorname{conv}(S))=\beta(S)$;

(h) $\beta\left(\bigcup_{|\lambda| \leq h} \lambda S\right)=h \beta(S)$.

The following result follows directly from the Hahn-Banach theorem.

Lemma 2.3 Let $E$ be a normed space with $x_{0} \neq 0$. Then there exists $\varphi \in E^{*}$ with $\|\varphi\|=1$ and $\varphi\left(x_{0}\right)=\left\|x_{0}\right\|$.

Definition 2.4 [16] Let $\alpha \geq 0$ and $f$ be a function defined on $[0,1]$. The fractional $q$-integral of the Riemann-Liouville type is $\left({ }_{R L} I_{q}^{0} f\right)(x)=f(x)$ and

$$
\left({ }_{R L} I_{q}^{\alpha} f\right)(x)=\int_{a}^{x} \frac{(x-q t)^{(\alpha-1)}}{\Gamma_{q}(\alpha)} f(t) d_{q} t, \quad \alpha \in \mathfrak{R}^{+}, x \in[0,1] .
$$

Definition 2.5 [14] The fractional $q$-derivative of the Riemann-Liouville type of order $\alpha \geq 0$ is defined by $\left({ }_{R L} D_{q}^{0} f\right)(x)=f(x)$ and

$$
\left({ }_{R L} D_{q}^{\alpha} f\right)(x)=\left(D_{q}^{[\alpha]} I_{q}^{[\alpha]-\alpha} f\right)(x), \quad \alpha>0,
$$

where $[\alpha]$ is the smallest integer greater than or equal to $\alpha$.

Definition 2.6 [14] The fractional $q$-derivative of the Caputo type of order $\alpha \geq 0$ is defined by

$$
\left({ }_{C} D_{q}^{\alpha} f\right)(x)=\left(I_{q}^{[\alpha]-\alpha} D_{q}^{[\alpha]} f\right)(x), \quad \alpha>0,
$$

where $[\alpha]$ is the smallest integer greater than or equal to $\alpha$.

Lemma 2.4 [14] Let $\alpha, \beta \geq 0$ and let $f$ be a function defined on $[0,1]$. Then the next formulas hold: 
(1) $\left(I_{q}^{\beta} I_{q}^{\alpha} f\right)(x)=\left(I_{q}^{\alpha+\beta} f\right)(x)$,

(2) $\left(D_{q}^{\beta} I_{q}^{\alpha} f\right)(x)=f(x)$.

Lemma 2.5 [32] Let D be a closed convex and equicontinuous subset of a metrizable locally convex vector space $C(J, E)$ such that $0 \in D$. Assume that $A: D \rightarrow D$ is weakly sequentially continuous. If the implication

$$
\bar{V}=\overline{\operatorname{conv}}(\{0\} \cup A(V)) \Rightarrow V \text { is relatively weakly compact, }
$$

holds for every subset $V$ of $D$, then $A$ has a fixed point.

\section{Main results}

Let us start by defining what we mean by a solution of the problem (1.1).

Definition 3.1 A function $u \in C\left(J, E_{\omega}\right)$ is said to be a solution of the problem (1.1) if $u$ satisfies the equation ${ }_{C} D_{q}^{\alpha} u+f(t, u)=0$ on $J$, and satisfy the conditions $u(0)=\left(D_{q}^{2} u\right)(0)=0$, $\gamma\left(D_{q} u\right)(1)+\beta D_{q}^{2} u(1)=0$.

For the existence results on the problem (1.1), we need the following auxiliary lemmas.

Lemma 3.1 [19] Let $\alpha>0$ and $n \in \mathbb{N}$. Then, the following equality holds:

$$
\left({ }_{R L} I_{q}^{\alpha} R L D_{q}^{n} f\right)(x)={ }_{R L} D_{q R L}^{n} I_{q}^{\alpha} f(x)-\sum_{k=0}^{\alpha-1} \frac{x^{\alpha-n+k}}{\Gamma_{q}(\alpha+k-n+1)}\left(D_{q}^{k} f\right)(0) .
$$

Lemma 3.2 [14] Let $\alpha>0$ and $n \in \mathfrak{R}^{+} \backslash \mathbb{N}$. Then the following equality holds:

$$
\left(I_{q}^{\alpha} C_{q}^{\alpha} f\right)(x)=f(x)-\sum_{k=0}^{[\alpha]-1} \frac{x^{k}}{\Gamma_{q}(k+1)}\left(D_{q}^{k} f\right)(0)
$$

We derive the corresponding Green's function for boundary value problem (1.1), which will play major role in our next analysis.

Lemma 3.3 Let $\rho \in C[0,1]$ be a given function, then the boundary-value problem

$$
\left\{\begin{array}{l}
{ }_{C} D_{q}^{\alpha} u+\rho(t)=0, \quad t \in[0,1], 2<\alpha \leq 3, \\
u(0)=\left(D_{q}^{2} u\right)(0)=0, \quad \gamma\left(D_{q} u\right)(1)+\beta D_{q}^{2} u(1)=0
\end{array}\right.
$$

has a unique solution

$$
u(t)=\int_{0}^{1} G(t, q s) \rho(s) d_{q} s,
$$

where $G(t, s)$ is defined by the formula

$$
G(t, s)= \begin{cases}\frac{t(1-s)^{(\alpha-2)}}{\Gamma_{q}(\alpha-1)}+\frac{\beta}{\gamma} \frac{t(1-s)^{(\alpha-3)}}{\Gamma_{q}(\alpha-2)}, & \text { if } 0 \leq t \leq s \leq 1, \\ \frac{t(1-s)^{(\alpha-2)}}{\Gamma_{q}(\alpha-1)}+\frac{\beta}{\gamma} \frac{t(1-s)^{(\alpha-3)}}{\Gamma_{q}(\alpha-2)}-\frac{(t-s)^{(\alpha-1)}}{\Gamma_{q}(\alpha)}, & \text { if } 0 \leq s \leq t \leq 1 .\end{cases}
$$

Here, $G(t, s)$ is called the Green's function of boundary value problem (3.1). 
Proof By Lemma 2.4 and Lemma 3.2, we can reduce the equation of problem (3.1) to an equivalent integral equation

$$
u(t)=u(0)+\frac{D_{q} u(0)}{\Gamma_{q}(2)} t+\frac{D_{q}^{2} u(0)}{\Gamma_{q}(3)} t^{2}-I_{q}^{\alpha} \rho(t)
$$

Applying the boundary conditions $u(0)=\left(D_{q}^{2} u\right)(0)=0$, we have

$$
u(t)=A t-\int_{0}^{t} \frac{(t-q s)^{(\alpha-1)}}{\Gamma_{q}(\alpha)} \rho(s) d_{q} s
$$

So, we have

$$
\begin{aligned}
& \left(D_{q} u\right)(t)=A-\int_{0}^{t} \frac{[\alpha-1]_{q}(t-q s)^{(\alpha-2)}}{\Gamma_{q}(\alpha)} \rho(s) d_{q} s, \\
& \left(D_{q}^{2} u\right)(t)=-\int_{0}^{t} \frac{[\alpha-1]_{q}[\alpha-2]_{q}(t-q s)^{(\alpha-3)}}{\Gamma_{q}(\alpha)} \rho(s) d_{q} s .
\end{aligned}
$$

Then, by the condition $\gamma\left(D_{q} u\right)(1)+\beta D_{q}^{2} u(1)=0$, we have

$$
A=\int_{0}^{1} \frac{(1-q s)^{(\alpha-2)}}{\Gamma_{q}(\alpha-1)} \rho(s) d_{q} s+\frac{\beta}{\gamma} \int_{0}^{1} \frac{(1-q s)^{(\alpha-3)}}{\Gamma_{q}(\alpha-2)} \rho(s) d_{q} s .
$$

Therefore, the unique solution of problem (3.1) is

$$
\begin{aligned}
u(t)= & A t-\int_{0}^{t} \frac{(t-q s)^{(\alpha-1)}}{\Gamma_{q}(\alpha)} \rho(s) d_{q} s \\
= & {\left[\int_{0}^{1} \frac{(1-q s)^{(\alpha-2)}}{\Gamma_{q}(\alpha-1)} \rho(s) d_{q} s+\frac{\beta}{\gamma} \int_{0}^{1} \frac{(1-q s)^{(\alpha-3)}}{\Gamma_{q}(\alpha-2)} \rho(s) d_{q} s\right] t } \\
& -\int_{0}^{t} \frac{(t-q s)^{(\alpha-1)}}{\Gamma_{q}(\alpha)} \rho(s) d_{q} s \\
= & \int_{0}^{1} G(t, q s) \rho(s) d_{q} s,
\end{aligned}
$$

which completes the proof.

Remark 3.1 From the expression of $G(t, s)$, it is obvious that $G(t, s)$ is continuous on $J \times J$.

Denote by

$$
G^{*}=\sup \left\{\int_{0}^{T}|G(t, s)| d_{q} s: t \in J\right\}
$$

To prove the main results, we need the following assumptions:

(H1) For each $t \in J$, the function $f(t, \cdot)$ is weakly sequentially continuous;

(H2) For each $x \in C(J, E)$, the function $f(\cdot, x(\cdot))$ is Pettis integrable on $J$;

(H3) There exists $p_{f} \in L^{\infty}\left(J, \mathbb{R}^{+}\right)$such that

$$
\|f(t, u)\| \leq p_{f}(t)\|u\| \quad \text { for a.e. } t \in J \text { and each } u \in E \text {; }
$$


$(\mathrm{H} 3)^{\prime}$ There exists $p_{f} \in L^{\infty}\left(J, \mathbb{R}^{+}\right)$and a continuous nondecreasing function $\psi:[0, \infty) \rightarrow$ $(0, \infty)$ such that

$$
\|f(t, u)\| \leq p_{f}(t) \psi(\|u\|) \quad \text { for a.e. } t \in J \text { and each } u \in E \text {; }
$$

(H4) For each bounded set $D \subset E$, and each $t \in J$, the following inequality holds:

$$
\beta(f(t, D)) \leq p_{f}(t) \cdot \beta(D)
$$

(H5) There exists a constant $R>0$ such that

$$
\frac{R}{\left\|p_{f}\right\|_{L^{\infty} \psi(R) G^{*}}}>1
$$

where $\left\|p_{f}\right\|_{L^{\infty}}=\sup \left\{p_{f}(t): t \in J\right\}$

Theorem 3.1 Let E be a reflexive Banach space and assume that (H1)-(H3) are satisfied.If

$$
\left\|p_{f}\right\|_{L^{\infty}} G^{*}<1
$$

then the problem (1.1) has at least one solution on J.

Proof Let the operator $\mathcal{A}: C(J, E) \rightarrow C(J, E)$ defined by the formula

$$
(\mathcal{A} u)(t):=\int_{0}^{1} G(t, q s) f(s, u(s)) d_{q} s,
$$

where $G(\cdot, \cdot)$ is the Green's function defined by (3.3). It is well known the fixed points of the operator $\mathcal{A}$ are solutions of the problem (1.1).

First notice that, for $x \in C(J, E)$, we have $f(\cdot, x(\cdot)) \in P(J, E)$ (assumption (H2)). Since, $s \mapsto$ $G(t, s) \in L^{\infty}(J)$, then $G(t, \cdot) f(\cdot, x(\cdot))$ is Pettis integrable for all $t \in J$ by Lemma 2.1 , and so the operator $\mathcal{A}$ is well defined.

Let $R>0$, and consider the set

$$
\begin{aligned}
D= & \left\{x \in C(J, E):\|x\|_{\infty} \leq R\right. \text { and } \\
& \left.\left\|x\left(t_{1}\right)-x\left(t_{2}\right)\right\| \leq R\left\|p_{f}\right\|_{L^{\infty}} \int_{0}^{1}\left|G\left(t_{2}, q s\right)-G\left(t_{1}, q s\right)\right| d_{q} s \text { for } t_{1}, t_{2} \in J\right\} .
\end{aligned}
$$

Clearly, the subset $D$ is closed, convex and equicontinuous. We shall show that $\mathcal{A}$ satisfies the assumptions of Lemma 2.5. The proof will be given in three steps.

Step 1: We will show that the operator $\mathcal{A}$ maps $D$ into itself.

Take $x \in D, t \in J$ and assume that $\mathcal{A} x(t) \neq 0$. Then there exists $\psi \in E^{*}$ such that $\|\mathcal{A} x(t)\|=$ $\psi(\mathcal{A} x(t))$. Thus,

$$
\begin{aligned}
\|(\mathcal{A} x)(t)\| & =\psi((\mathcal{A} x)(t))=\int_{0}^{1} G(t, q s) f(s, y(s)) d_{q} s \\
& \leq \int_{0}^{1}|G(t, q s)| \cdot \psi(f(s, x(s))) d_{q} s
\end{aligned}
$$




$$
\begin{aligned}
& \leq \int_{0}^{1}|G(t, q s)| \cdot p_{f}(s) \cdot\|x(s)\| d_{q} s \\
& \leq\left\|p_{f}\right\|_{L^{\infty}} R G^{*} \\
& \leq R .
\end{aligned}
$$

Let $\tau_{1}, \tau_{2} \in J, \tau_{1}<\tau_{2}$ and $\forall x \in D$, so $\mathcal{A} x\left(\tau_{2}\right)-\mathcal{A} x\left(\tau_{1}\right) \neq 0$. Then there exists $\psi \in E^{*}$, such that $\left\|\mathcal{A} x\left(\tau_{2}\right)-\mathcal{A} x\left(\tau_{1}\right)\right\|=\psi\left(\mathcal{A} x\left(\tau_{2}\right)-\mathcal{A} x\left(\tau_{1}\right)\right)$. Hence,

$$
\begin{aligned}
\left\|\mathcal{A} x\left(\tau_{2}\right)-\mathcal{A} x\left(\tau_{1}\right)\right\| & =\int_{0}^{1}\left[G\left(\tau_{2}, q s\right)-G\left(\tau_{1}, q s\right)\right] \cdot f(s, x(s)) d_{q} s \\
& \leq \int_{0}^{1}\left|G\left(\tau_{2}, q s\right)-G\left(\tau_{1}, q s\right)\right| \cdot\|f(s, x(s))\| d_{q} s \\
& \leq R\left\|p_{f}\right\|_{L^{\infty}} \int_{0}^{1}\left|G\left(\tau_{2}, q s\right)-G\left(\tau_{1}, q s\right)\right| d_{q} s,
\end{aligned}
$$

this means that $\mathcal{A}(D) \subset D$.

Step 2: We will show that the operator $\mathcal{A}$ is weakly sequentially continuous.

Let $\left(x_{n}\right)$ be a sequence in $D$ and let $\left(x_{n}(t)\right) \rightarrow x(t)$ in $(E, w)$ for each $t \in J$. Fix $t \in J$. Since $f$ satisfies assumptions (H1), we have $f\left(t, x_{n}(t)\right)$ converge weakly uniformly to $f(t, x(t))$. Hence, the Lebesgue dominated convergence theorem for Pettis integrals implies $\mathcal{A} x_{n}(t)$ converges weakly uniformly to $\mathcal{A} x(t)$ in $E_{\omega}$. Repeating this for each $t \in J$ shows $\mathcal{A} x_{n} \rightarrow \mathcal{A} x$. Then $\mathcal{A}: D \rightarrow D$ is weakly sequentially continuous.

Step 3: The implication (2.1) holds. Now let $V$ be a subset of $D$ such that $V \subset$ $\overline{\operatorname{conv}}(\mathcal{A}(V) \cup\{0\})$. Clearly, $V(t) \subset \overline{\operatorname{conv}}(\mathcal{A}(V) \cup\{0\})$ for all $t \in J$. Hence, $\mathcal{A} V(t) \subset \mathcal{A} D(t)$, $t \in J$, is bounded in $E$. Thus, $\mathcal{A} V(t)$ is weakly relatively compact since a subset of a reflexive Banach space is weakly relatively compact if and only if it is bounded in the norm topology. Therefore,

$$
\begin{aligned}
v(t) & \leq \beta(\mathcal{A}(V)(t) \cup\{0\}) \\
& \leq \beta(\mathcal{A}(V)(t)) \\
& =0,
\end{aligned}
$$

thus, $V$ is relatively weakly compact in $E$. In view of Lemma 2.5 , we deduce that $\mathcal{A}$ has a fixed point, which is obviously a solution of the problem (1.1). This completes the proof.

Remark 3.2 In Theorem 3.1, we presented an existence result for weak solutions of the problem (1.1) in the case where the Banach space $E$ is reflexive. However, in the nonreflexive case, conditions $(\mathrm{H} 1)-(\mathrm{H} 3)$ are not sufficient for the application of Lemma 2.5; the difficulty is with condition (2.1).

Theorem 3.2 Let E be a Banach space, and assume assumptions (H1), (H2), (H3), (H4) are satisfied.If (3.9) holds, then the problem (1.1) has at least one solution on J.

Theorem 3.3 Let E be a Banach space, and assume assumptions (H1), (H2), (H3)', (H4), (H5) are satisfied. If (3.9) holds, then the problem (1.1) has at least one solution on J. 
Proof Assume that the operator $\mathcal{A}: C(J, E) \rightarrow C(J, E)$ is defined by the formula (3.10). It is well known the fixed points of the operator $\mathcal{A}$ are solutions of the problem (1.1).

First notice that, for $x \in C(J, E)$, we have $f(\cdot, x(\cdot)) \in P(J, E)$ (assumption (H2)). Since, $s \mapsto$ $G(t, s) \in L^{\infty}(J)$, then $G(t, \cdot) f(\cdot, x(\cdot))$ for all $t \in J$ is Pettis integrable (Lemma 2.1), and thus, the operator $\mathcal{A}$ makes sense.

Let $R>0$, and consider the set

$$
\begin{aligned}
\mathcal{D}= & \left\{x \in C(J, E):\|x\|_{\infty} \leq R\right. \text { and } \\
& \left.\left\|x\left(t_{1}\right)-x\left(t_{2}\right)\right\| \leq\left\|p_{f}\right\|_{L^{\infty}} \psi(R) \int_{0}^{1}\left|G\left(t_{2}, q s\right)-G\left(t_{1}, q s\right)\right| d_{q} s \text { for } t_{1}, t_{2} \in J\right\},
\end{aligned}
$$

clearly, the subset $\mathcal{D}$ is closed, convex and equicontinuous. We shall show that $\mathcal{A}$ satisfies the assumptions of Lemma 2.5. The proof will be given in three steps.

Step 1: We will show that the operator $\mathcal{A}$ maps $\mathcal{D}$ into itself.

Take $x \in \mathcal{D}, t \in J$ and assume that $\mathcal{A} x(t) \neq 0$. Then there exists $\psi \in E^{*}$ such that $\|\mathcal{A} x(t)\|=$ $\psi(\mathcal{A} x(t))$. Thus,

$$
\begin{aligned}
\|(\mathcal{A} x)(t)\| & =\psi((\mathcal{A} x)(t))=\psi\left(\int_{0}^{1} G(t, q s) f(s, y(s)) d_{q} s\right) \\
& \leq \int_{0}^{T}|G(t, q s)| \cdot \psi(f(s, x(s))) d_{q} s \\
& \leq \int_{0}^{T}|G(t, q s)| \cdot p_{f}(s) \cdot \psi(\|x(s)\|) d_{q} s \\
& \leq\left\|p_{f}\right\|_{L^{\infty}} \psi(R) G^{*} \\
& \leq R .
\end{aligned}
$$

Let $\tau_{1}, \tau_{2} \in J, \tau_{1}<\tau_{2}$ and $\forall x \in \mathcal{D}$, so $\mathcal{A} x\left(\tau_{2}\right)-\mathcal{A} x\left(\tau_{1}\right) \neq 0$. Then there exist $\psi \in E^{*}$ such that

$$
\left\|\mathcal{A} x\left(\tau_{2}\right)-\mathcal{A} x\left(\tau_{1}\right)\right\|=\psi\left(\mathcal{A} x\left(\tau_{2}\right)-\mathcal{A} x\left(\tau_{1}\right)\right) .
$$

Thus,

$$
\begin{aligned}
\left\|\mathcal{A} x\left(\tau_{2}\right)-\mathcal{A} x\left(\tau_{1}\right)\right\| & =\psi\left(\int_{0}^{1}\left[G\left(\tau_{2}, q s\right)-G\left(\tau_{1}, q s\right)\right] \cdot f(s, x(s)) d_{q} s\right) \\
& \leq \int_{0}^{1}\left|G\left(\tau_{2}, q s\right)-G\left(\tau_{1}, q s\right)\right| \cdot\|f(s, x(s))\| d_{q} s \\
& \leq \psi(R)\left\|p_{f}\right\|_{L^{\infty}} \int_{0}^{1}\left|G\left(\tau_{2}, q s\right)-G\left(\tau_{1}, q s\right)\right| d_{q} s
\end{aligned}
$$

this means that $\mathcal{A}(\mathcal{D}) \subset \mathcal{D}$.

Step 2: We will show that the operator $\mathcal{A}$ is weakly sequentially continuous.

Let $\left(x_{n}\right)$ be a sequence in $\mathcal{D}$ and let $\left(x_{n}(t)\right) \rightarrow x(t)$ in $(E, w)$ for each $t \in J$. Fix $t \in J$. Since $f$ satisfies assumptions (H1), we have $f\left(t, x_{n}(t)\right)$, converging weakly uniformly to $f(t, x(t))$. Hence, the Lebesgue dominated convergence theorem for Pettis integral implies $\mathcal{A} x_{n}(t)$ 
converging weakly uniformly to $\mathcal{A} x(t)$ in $E_{\omega}$. We do it for each $t \in J$ so $\mathcal{A} x_{n} \rightarrow \mathcal{A} x$. Then $\mathcal{A}: \mathcal{D} \rightarrow \mathcal{D}$ is weakly sequentially continuous.

Step 3: The implication (2.1) holds. Now let $V$ be a subset of $\mathcal{D}$ such that $V \subset$ $\overline{\operatorname{conv}}(\mathcal{A}(V) \cup\{0\})$. Clearly, $V(t) \subset \overline{\operatorname{conv}}(\mathcal{A}(V) \cup\{0\})$ for all $t \in J$. Hence, $\mathcal{A} V(t) \subset \mathcal{A D}(t)$, $t \in J$, is bounded in $E$. Using this fact, assumption (H4), Lemma 2.2 and the properties of the measure $\beta$, we have for each $t \in J$

$$
\begin{aligned}
v(t) & \leq \beta(\mathcal{A}(V)(t) \cup\{0\}) \\
& \leq \beta(\mathcal{A}(V)(t)) \\
& =\beta\left\{\int_{0}^{1} G(t, q s) f(s, V(s)) d_{q} s\right\} \\
& \leq \int_{0}^{1}|G(t, q s)| \cdot p_{f}(s) \cdot \beta(V(s)) d_{q} s \\
& \leq\left\|p_{f}\right\|_{L^{\infty}} \cdot \int_{0}^{1}|G(t, q s)| \cdot v(s) d_{q} s \\
& \leq\left\|p_{f}\right\|_{L^{\infty}} \cdot\|v\|_{\infty} \cdot G^{*},
\end{aligned}
$$

which gives

$$
\|v\|_{\infty} \leq\left\|p_{f}\right\|_{L^{\infty}} \cdot\|v\|_{\infty} \cdot G^{*}
$$

This means that

$$
\|v\|_{\infty} \cdot\left[1-\left\|p_{f}\right\|_{L^{\infty}} \cdot G^{*}\right] \leq 0
$$

By (3.9), it follows that $\|v\|_{\infty}=0$, that is $v(t)=0$ for each $t \in J$, and then $V(t)$ is relatively weakly compact in $E$. In view of Lemma 2.5 , we deduce that $\mathcal{A}$ has a fixed point which is obviously a solution of the problem (1.1). This completes the proof.

\section{Competing interests}

The authors declare that they have no competing interests.

\section{Authors' contributions}

All authors contributed equally to the manuscript. All authors read and approved the final manuscript.

\section{Acknowledgements}

Dedicated to Professor Hari M Srivastava.

This research was supported by the National Natural Science Foundation of China $(11161027,11262009)$. The authors are thankful to the referees for their careful reading of the manuscript and insightful comments.

Received: 10 December 2012 Accepted: 4 April 2013 Published: 19 April 2013

\section{References}

1. Podlubny, I: Fractional Differential Equations. Academic Press, San Diego (1999)

2. Kilbas, AA, Srivastava, HM, Trujillo, Jj: Theory and Applications of Fractional Differential Equations. North-Holland Mathematical Studies, vol. 204. Elsevier, Amsterdam (2006)

3. Srivastava, HM: Some generalizations and basic (or q-) extensions of the Bernoulli, Euler and Genocchi polynomials. Appl. Math. Inf. Sci. 5(3), 390-444 (2011)

4. Srivastava, HM, Choi, J: Zeta and q-Zeta Functions and Associated Series and Integrals. Elsevier, Amsterdam (2012)

5. El-Shahed, M: Existence of solution for a boundary value problem of fractional order. Adv. Appl. Math. Anal. 2(1), 1-8 (2007)

6. Zhang, S: Existence of solution for a boundary value problem of fractional order. Acta Math. Sci. 26(2), 220-228 (2006) 
7. El-Shahed, M, Al-Askar, FM: On the existence of positive solutions for a boundary value problem of fractional order. Int. J. Math. Anal. 4(13-16), 671-678 (2010)

8. Bai, Z, Lü, H: Positive solutions for boundary value problem of nonlinear fractional differential equation. J. Math. Anal. Appl. 311(2), 495-505 (2005)

9. Zhou, W, Chu, Y: Existence of solutions for fractional differential equations with multi-point boundary conditions. Commun. Nonlinear Sci. Numer. Simul. 17(3), 1142-1148 (2012)

10. Zhou, W, Peng, J, Chu, Y: Multiple positive solutions for nonlinear semipositone fractional differential equations. Discrete Dyn. Nat. Soc. 2012, Article ID 850871 (2012). doi:10.1155/2012/850871

11. Jackson, FH: On q-functions and a certain difference operator. Trans. R. Soc. Edinb. 46, $253-281$ (1908)

12. Jackson, R: On q-definite integrals. Q. J. Pure Appl. Math. 41, 193-203 (1910)

13. Kac, V, Cheung, P: Quantum Calculus. Springer, New York (2002)

14. Stanković, MS, Rajković, PM, Marinković, SD: On q-fractional derivatives of Riemann-Liouville and Caputo type. http://arxiv.org/abs/0909.0387 (2009)

15. Al-Salam, WA: Some fractional $q$-integrals and q-derivatives. Proc. Edinb. Math. Soc. 15(2), 135-140 (1967)

16. Agarwal, RP: Certain fractional $q$-integrals and q-derivatives. Math. Proc. Camb. Philos. Soc. 66, 365-370 (1969)

17. Rajković, PM, Marinković, SD, Stanković, MS: On q-analogues of Caputo derivative Mittag-Leffler function. Fract. Calc. Appl. Anal. 10(4), 359-373 (2007)

18. El-Shahed, M, Hassan, HA: Positive solutions of q-difference equation. Proc. Am. Math. Soc. 138(5), 1733-1738 (2010)

19. Ferreira, RAC: Nontrivial solutions for fractional $q$-difference boundary value problems. Electron. J. Qual. Theory Differ. Equ. 2010, 70 (2010)

20. Ferreira, RAC: Positive solutions for a class of boundary value problems with fractional $q$-differences. Comput. Math. Appl. 61(2), 367-373 (2011)

21. El-Shahed, M, Al-Askar, FM: Positive solutions for boundary value problem of nonlinear fractional $q$-difference equation. ISRN Math. Anal. 2011, Article ID 385459 (2011). doi:10.5402/2011/385459

22. Ahmad, B, Alsaedi, A, Ntouyas, SK: A study of second-order q-difference equations with boundary conditions. Adv. Differ. Equ. 2012, 35 (2012). doi:10.1186/1687-1847-2012-35

23. Ahmad, B, Nieto, Jj: On nonlocal boundary value problems of nonlinear q-difference equations. Adv. Differ. Equ. 2012, 81 (2012). doi:10.1186/1687-1847-2012-81

24. Banaś, J, Goebel, K: Measures of Noncompactness in Banach Spaces. Dekker, New York (1980)

25. Banaś, J, Sadarangani, K: On some measures of noncompactness in the space of continuous functions. Nonlinear Anal. 68(2), 377-383 (2008)

26. Guo, D, Lakshmikantham, V, Liu, X: Nonlinear Integral Equations in Abstract Spaces, vol. 373. Kluwer Academic, Dordrecht (1996)

27. Krzyska, S, Kubiaczyk, l: On bounded pseudo and weak solutions of a nonlinear differential equation in Banach spaces. Demonstr. Math. 32(2), 323-330 (1999)

28. Lakshmikantham, V, Leela, S: Nonlinear Differential Equations in Abstract Spaces, vol. 2. Pergamon, Oxford (1981)

29. Mönch, $\mathrm{H}$ : Boundary value problems for nonlinear ordinary differential equations of second order in Banach spaces. Nonlinear Anal. 4(5), 985-999 (1980)

30. O'Regan, D: Fixed point theory for weakly sequentially continuous mapping. Math. Comput. Model. 27(5), 1-14 (1998)

31. O'Regan, D: Weak solutions of ordinary differential equations in Banach spaces. Appl. Math. Lett. 12(1), 101-105 (1999)

32. Szufla, S: On the application of measure of noncompactness to existence theorems. Rend. Semin. Mat. Univ. Padova 75, 1-14 (1986)

33. Szufla, S, Szukala, A: Existence theorems for weak solutions of $n$th order differential equations in Banach spaces. Funct. Approx. Comment. Math. 26, 313-319 (1998). Dedicated to Julian Musielak

34. Salem, HAH: On the fractional order m-point boundary value problem in reflexive Banach spaces and weak topologies. J. Comput. Appl. Math. 224(2), 565-572 (2009)

35. Salem, HAH, El-Sayed, AMA, Moustafa, OL: A note on the fractional calculus in Banach spaces. Studia Sci. Math. Hung. 42(2), 115-130 (2005)

36. Benchohra, M, Graef, JR, Mostefai, FZ: Weak solutions for nonlinear fractional differential equations on reflexive Banach spaces. Electron. J. Qual. Theory Differ. Equ. 2010, 54 (2010)

37. Zhou, W, Chang, Y, Liu, H: Weak solutions for nonlinear fractional differential equations in Banach spaces. Discrete Dyn. Nat. Soc. 2012, Article ID 527969 (2012). doi:10.1155/2012/527969

38. Zhou, W, Liu, H: Existence of weak solutions for nonlinear fractional differential inclusion with non-separated boundary conditions. J. Appl. Math. 2012, Article ID 530624 (2012). doi:10.1155/2012/530624

39. Pettis, BJ: On integration in vector spaces. Trans. Am. Math. Soc. 44(2), 277-304 (1938)

40. De Blasi, FS: On the property of the unit sphere in a Banach space. Bull. Math. Soc. Sci. Math. Répub. Social. Roum. 21(3-4), 259-262 (1977)

doi:10.1186/1687-1847-2013-113

Cite this article as: Zhou and Liu: Existence solutions for boundary value problem of nonlinear fractional

q-difference equations. Advances in Difference Equations 2013 2013:113. 